\title{
Colossal magnetoresistance oxides: phenomena and questions
}

\author{
B Y T. V. RAMAKRISHNAN \\ Department of Physics, Indian Institute of Science, \\ Bangalore 560012, India
}

Some unusual electronic properties of doped manganites are described. The lowtemperature resistivity of the metallic ferromagnet has a large $T^{2}$ term. The residual resistivity can be much larger than the Mott maximum, and depends exponentially on spontaneous magnetization. There is much evidence for strong spatial and temporal fluctuation effects. The available theories are discussed in the light of these phenomena, and it is suggested that a novel localizing mechanism is at work, leading to extreme sensitivity of physical properties to small atomic changes.

Keywords: colossal magnetoresistance; residual resistivity; fluctuation effects; oxygen isotope effect; charge ordering; optical conductivity

\section{Introduction}

The discovery of colossal magnetoresistance in mixed valent manganites (for a recent review, see Rao et al. 1998; see also Coey \& von Molnar 1998) has led to a resurgence of interest in this family of compounds. Many spectacular and unusual new phenomena have been discovered, raising basic questions about the nature of electronic states and phases. Many of the observed effects are due to the strong connection between magnetism and the insulator to metal transition special to these systems. In the spirit of this meeting, I will describe some of the observations, and point out the questions they raise. While a general set of ideas exists, the theoretical explanations seem inadequate.

After a brief introduction, I focus on anomalous electrical transport behaviour of the 'metallic' manganites. Evidence for large fluctuation effects, from magnetic and optical measurements, is also presented. Charge ordering in manganites is briefly mentioned. Ideas and theories are discussed in the perspective of these phenomena.

The stoichiometric manganites are antiferromagnetic insulators with a slightly distorted perovskite structure. In $\mathrm{LaMnO}_{3}$, a typical member, the $\mathrm{Mn}^{3+}$ has octahedral coordination, and its four d electrons occupy $t_{2 g}$ and $e_{g}$ levels. Because of strong Hund's rule effects, the ground state has the highest spin possible, namely $S=2$, with three electrons in the triply degenerate $t_{2 g}$ orbitals and one in the higher, doubly degenerate $e_{g}$ orbital. The double degeneracy of the latter is split by a Jahn-Teller distortion. $\mathrm{LaMnO}_{3}$ is a Mott insulator, with one $e_{g}$ like d electron per unit cell or a half-filled band, and a large Mott-Hubbard $U$, of about $5 \mathrm{eV}$ or more. The antiferromagnetism is due to $\mathrm{Mn}^{3+}-\mathrm{Mn}^{3+}$ superexchange mediated by hybridization with oxygen p orbitals $\left(\mathrm{LaMnO}_{3}\right.$ is ferromagnetic in plane, with opposite spin nearest neighbour planes).

On substituting trivalent La with a divalent ion such as $\mathrm{Ca}$ or $\mathrm{Sr}$, one d electron

Phil. Trans. R. Soc. Lond. A (1998) 356, 41-55

(C) 1998 The Royal Society

Printed in Great Britain

TEX Paper 


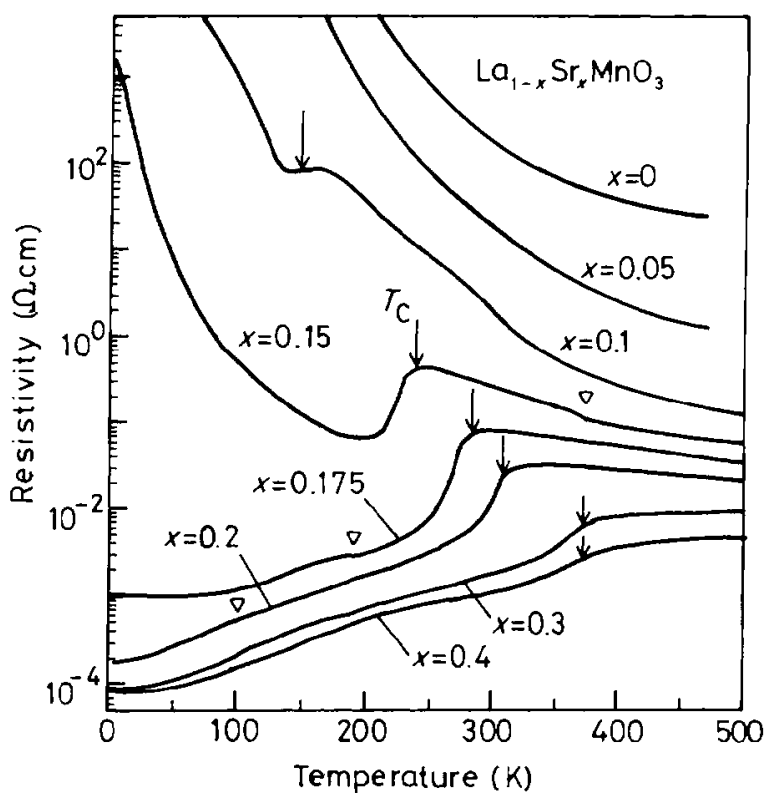

Figure 1. The resistivity of single crystal $\mathrm{La}_{1-x} \mathrm{Sr}_{x} \mathrm{MnO}_{3}$ as a function of temperature.

is transferred out from $\mathrm{Mn}^{4+}$ to oxygen, so that $\mathrm{La}_{1-x} \mathrm{Sr}_{x} \mathrm{MnO}_{3}$, for instance, has a fraction $(1-x)$ of the $\mathrm{Mn}$ ions in the $4^{+}$state and fraction $x$ in the $3^{+}$state. This mixed valent crystal is metallic because the $e_{g}$ electron can hop from an $\mathrm{Mn}^{4+}$ site to a neighbouring $\mathrm{Mn}^{3+}$ site. Because of the strong Hund's rule coupling, the hopping is proportional to the probability amplitude that the initial and final states have the maximum spin $S=2$. Thus, the $e_{g}$ electron kinetic energy favours parallel or ferromagnetic orientation of nearest neighbour spins. This is the double exchange of Zener (1951), and of Anderson \& Hasegawa (1955). The ground state of $\mathrm{La}_{1-x} \mathrm{Sr}_{x} \mathrm{MnO}_{3}$ is expected to be a ferromagnetic metal, because of the kinetic energy gained by the $e_{g}$ electron moving parallel to the aligned $t_{2 g}$ spins. On increasing the temperature, the 'resident' $t_{2 g}$ spins disorder because of entropic reasons, the $e_{g}$ electron does not propagate well, and there is transition to a paramagnetic insulator phase. The moving $e_{g}$ electron carries its Jahn-Teller distortion with it; this polaronic effect aids the transition or may even be the main cause.

The ideas mentioned, namely mixed valence of $\mathrm{Mn}$, double exchange and JahnTeller distortion related polaronic effects, seem appropriate at least qualitatively. For example, $\mathrm{La}_{1-x} \mathrm{Sr}_{x} \mathrm{MnO}_{3}$ shows a paramagnet to ferromagnet transition for $x>0.17$, with a $T_{\mathrm{c}}$ in the range $200-300 \mathrm{~K}$ and nearly coinciding with it an insulator to metal transition (figure 1) (Urushibara et al. 1995). There is considerable structural evidence for polaronic effects. The colossal magnetoresistance that is observed near the transition can be rationalized as being due to the fact that a magnetic field, by partially aligning moments, makes it easier for electrons to move. This effect is strongest near the transition where the magnetic susceptibility is the largest. Theories incorporating some of the above ideas have been developed.

It becomes clear, however, that many significant features of the transition are not well explained. The so-called metallic state often has resistivities such that the electron mean-free path is much smaller than a lattice spacing. This is true even in the best prepared single crystalline or thin film samples, say near $T_{\mathrm{c}}$. This is 


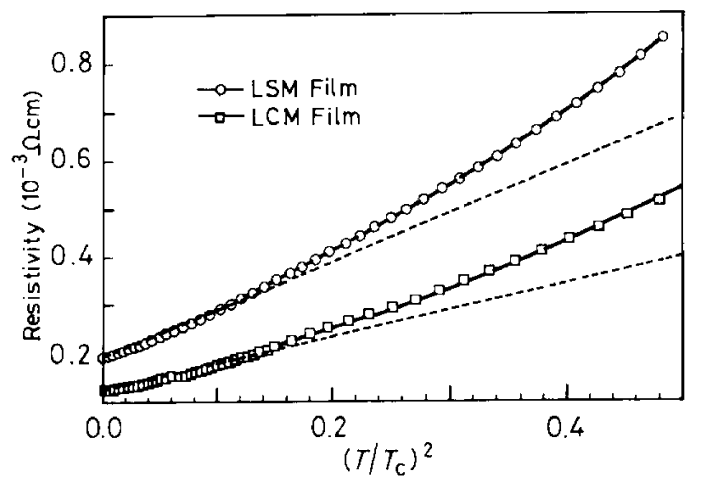

Figure 2. The low-temperature resistivity of $\mathrm{La}_{0.67}(\mathrm{Sr} / \mathrm{Ca})_{0.33} \mathrm{MnO}_{3}$ films. The solid lines are a fit to $R_{0}+R_{2} T^{2}+R_{9 / 2} T^{9 / 2}$. The dotted line is the best fit to a constant plus a quadratic term (from Snyder et al. 1996).

uncommon, though possible if $e_{g}$ electrons hop largely inelastically. The size of the colossal magnetoresistance is quite unusual. A magnetic field of a few teslas (energy of a few K) makes a large difference to electronic states and transport at a temperature of a few hundred degrees, which is itself more than an order of magnitude smaller than the electron kinetic energy $(1 \mathrm{eV})$. Another curious feature is that the paramagnetic to ferromagnetic transition generally takes place below (often about $50 \mathrm{~K}$ below) the insulator-metal transition, defined as the temperature where the electrical resistivity has a maximum.

I now describe several other observations, point out how they are surprising, describe the theoretical ideas developed and try to compare the two.

\section{Transport properties}

\section{(a) Low temperature resistivity}

The ferromagnetic metallic state of the optimally doped $\mathrm{La}_{2-x} \mathrm{~S}_{r_{x}} \mathrm{CaMnO}_{3}(x \sim$ 0.3 ), in high-quality single crystals as well as thin films, has a residual resistivity as small as $100 \mu \Omega \mathrm{cm}$, corresponding to a mean-free path of order $40-60 \AA$. The resistivity increases very rapidly with temperature (figure 2) (Snyder et al. 1996). The increase can be fitted to the dependence

$$
\rho(T)=\rho_{0}+A T^{2}+B T^{9 / 2},
$$

where the $T^{2}$ term is much the bigger one. If the ground state is fully magnetized, with all spins pointing up, single spin wave processes (figure $3 a$ ) have very little weight since there is an exponentially low density of final down spin electron states. The leading current relaxing process at low temperatures involves two spin waves (figure $3 b$ ) and depends on temperature as $T^{9 / 2}$ (Kubo \& Ohata 1972). This is not experimentally the leading term, which goes as $T^{2}$ and is very large. The $T^{2}$ term, if extrapolated, would lead to nearly the Mott maximum value resistivity for a temperature of order the ferromagnetic Curie temperature $T_{\mathrm{c}}$ ! Such a large $T^{2}$ term is very uncommon. At $T \simeq \frac{1}{2} T_{\mathrm{c}}$, the mean-free path has decreased from about $60 \AA$ to about $6-10 \AA$.

It is clear from the above that the picture of the low-temperature phase as an effectively non-interacting fully spin polarized electron system with spin waves as the

Phil. Trans. R. Soc. Lond. A (1998) 


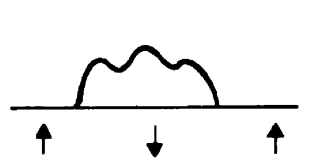

(a)

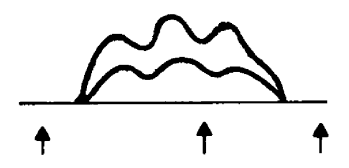

(b)

Figure 3. Spin wave scattering processes contributing to low-temperature resistivity in a ferromagnetic metal. (a) Single spin flip process, possible if both up and down spin electron densities are non-zero. (b) The leading two spin wave, process for a fully polarized metal.
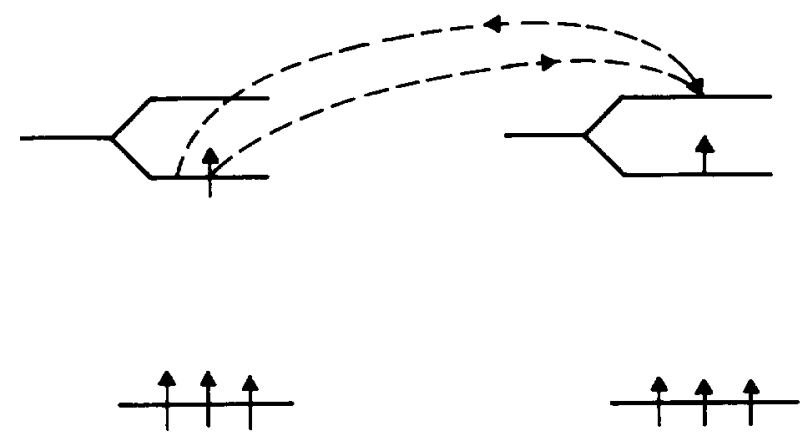

Figure 4. A possible nearest-neighbour virtual hopping process that leads to a nearest-neighbour attractive interaction.

elementary excitations is inadequate even when the ground state is very well ordered magnetically and spin waves are well-defined low-energy magnetic excitations. One can certainly imagine interactions arising out of nearest-neighbour hopping terms in higher order; one such is shown in figure 4 . It is a ferromagnetic nearest-neighbour attraction $-V_{i j} n_{i} n_{j}$. It lowers the energy of the state with one $e_{g}$ electron on site $i$ and another on site $j$, in the spin configuration shown, for example. Whether this interaction is strong enough to cause a $T^{2}$ resistivity of the observed size is not clear.

It needs to be pointed out that low-temperature specific heat (Hamilton et al. 1996; Coey et al. 1995; Okimoto et al. 1997) of metallic manganites shows an electronic linear term close to what is expected from a tight binding band; there is no strong enhancement of the linear specific heat while the $T^{2}$ coefficient of resistivity is highly enhanced, approximately by the ratio $T_{\mathrm{F}} / T_{\mathrm{c}}\left(\Delta \rho(T) \sim \rho_{\text {Mott }}\left(T / T_{\mathrm{c}}\right)^{2} !\right)$. This is in striking contrast to systems near the Mott transition where both $\gamma$ and $\Delta \rho(T)$ are enhanced similarly.

\section{Residual resistivity}

As mentioned above, in clean metallic manganites, the residual resistivity can be quite small, smaller by a factor of ten or more than the maximum metallic value of about $1-2 \mathrm{~m} \Omega \mathrm{cm}$. However, many polycrystalline systems with nearly optimum size of the rare earth (or substituent) ions, as well as systems with non-optimal (generally smaller) ion size, show extremely high values of residual resistivity. These values can be several orders of magnitude higher than the maximum metallic resistivity, and yet the resistivity seems to flatten out at $T \rightarrow 0$ without increasing to infinity as it should for an insulator. This is in contrast to the behaviour of all other strongly disordered and or correlated systems in which as a function of disorder or correlation, a maximum metallic resistivity (the Mott value corresponding to $k_{\mathrm{F}} l \sim 1$ ) separates

Phil. Trans. R. Soc. Lond. A (1998) 


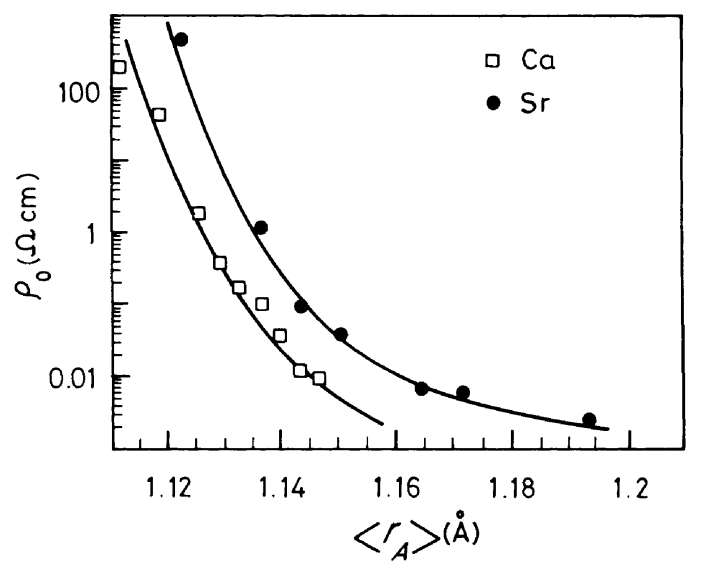

Figure 5. The residual resistivity of $\left(\mathrm{RE}_{1-x} \mathrm{RE}_{x}^{\prime}\right)_{2 / 3} \mathrm{~A}_{1 / 3} \mathrm{MnO}_{3}$ ceramic samples shown as a function of mean $\mathrm{A}$ site radius $\left\langle r_{\mathrm{A}}\right\rangle$ for two different alkaline earth ions $\mathrm{A}=\mathrm{Ca}$, $\mathrm{Sr}$ (from Garcia-Muñoz et al. 1997).

the disordered metal from the insulator. There are scale-dependent localization effects near critical disorder due to which, at very low temperatures, the conductivity goes smoothly to zero as the disorder approaches a critical value; however, on the large experimental scales of temperature and disorder in most disordered systems these quantum critical effects are invisible and the Mott dividing line is very real indeed.

One example of the spectacular violation of the Mott limit is the resistivity of $\mathrm{RE}_{0.7} \mathrm{~A}_{0.3} \mathrm{MnO}_{3}$ films measured by Coey et al. (1995), where the lanthanide RE is either $\mathrm{Nd}$ or $\mathrm{La}$, and the alkaline earth ion $\mathrm{A}$ is either $\mathrm{Sr}$ or $\mathrm{Ba}$. The $\mathrm{NdSrMnO}_{3}$ sample has a residual resistivity of $10^{4} \Omega \mathrm{cm}$, and a ferromagnetic ordering temperature of about $100 \mathrm{~K}$, while $\mathrm{LaSrMnO}_{3}$ has a resistivity of about $10^{-4} \Omega \mathrm{cm}$ and a $T_{\mathrm{c}}$ close to $300 \mathrm{~K}$. The two systems have the same carrier density. $\mathrm{Nd}^{3+}$ is much smaller than $\mathrm{La}^{3+}$, and this can cause various structural and electronic effects. A common way of parametrizing the structure is through the composition averaged radius $(1-x) r_{\mathrm{RE}}+x r_{\mathrm{A}}=\left\langle r_{\mathrm{A}}\right\rangle$. An exponential variation of $\rho_{0}$ with $\left\langle r_{\mathrm{A}}\right\rangle$ has been noted by Mahesh et al. (1995b). For ceramic samples with $x=0.3$, in which $\left\langle r_{\mathrm{A}}\right\rangle$ is varied by mixing two different rare earth ions, keeping the total fraction the same (i.e. 0.7) per formula unit, Garcia-Munoz et al. (1997) obtain a dependence of residual resistivity on $\left\langle r_{\mathrm{A}}\right\rangle$ shown in figure 5 . We note that the residual resistivity can be as much as $10^{6}$ times larger than the maximum metallic value!

This is certainly unique and quite astonishing, because in most disordered systems the Mott criterion is well obeyed experimentally (within factors of two), the main reason being its geometrical nature. It is based on the equality of two lengths, namely the elastic scattering mean-free path and the de Broglie wavelength of the electron. As such, it is independent of the bandwidth. This is easily seen in a tight binding model on a lattice of spacing $a$. In three dimensions, the minimum metallic conductivity is $e^{2} / \hbar a$ and does not depend on the bandwidth $|z t|$ (it depends weakly on band filling). It can also be shown, at least within perturbation theory, that quasi-particle renormalization (due to frequency-dependent self energy) has no effect on the residual Boltzmann conductivity. Thus it is difficult to sustain the common belief that polaronic reduction in bandwidth due to decreasing $\left\langle r_{\mathrm{A}}\right\rangle$ is the cause of the enormous residual resistivities observed. Moreover, the reduction in bandwidth with a few percent change in $\left\langle r_{\mathrm{A}}\right\rangle$ is not normally expected to be very large.

Phil. Trans. R. Soc. Lond. A (1998) 
If the effective bandwidth $W_{\text {eff }}$ due to $\mathrm{J}-\mathrm{T}$ polaron formation is very small, one expects the following temperature dependence for the conductivity. At $T \gg W_{\text {eff }}$, one has localized polarons hopping incoherently from site to site. If the intersite hopping probability is $p_{i}$, the conductivity of this classical gas is $\sigma \sim p_{i} / k_{\mathrm{B}} T$. If the intersite hopping is not activated, $p_{i}$ does not depend on temperature. Thus the conductivity increases with decreasing temperature, and at $T \sim W_{\text {eff }}$, goes over to the tight binding Mott value $e^{2} / \hbar a$. (Crudely, $p_{i} \geqslant W_{\text {eff }}$ so that the conductivity at $T \simeq W_{\text {eff }}$ is independent of the latter.) This is not the observed behaviour. Other purely thermally activated hopping processes give exponentially increasing resistivity with decreasing temperature, the exponent depending on the actual process (variable range hopping, etc.).

The following observations are suggestive as to the possible causes of the large residual resistivity.

The reduction in the mean rare earth ionic radius is accompanied by a bending of the $\mathrm{Mn}-\mathrm{O}-\mathrm{Mn}$ bond, from 170 to $155^{\circ}$ as $r_{\mathrm{A}}$ decreases from 1.20 to $1.13 \AA$. This implies that there is an increasing antiferromagnetic component to the $\mathrm{Mn}-\mathrm{Mn}$ spin coupling, which is reflected in the rather rapid decrease in the saturation magnetization as well as $T_{\mathrm{c}}$ with $r_{\mathrm{A}}$ (Garcia-Muñoz et al. 1997). Thus there is magnetic frustration and the energetically favourable arrangement is not one in which all $\mathrm{Mn}$ spins are parallel to each other.

The consequences of possible magnetic frustration for transport can be phenomenologically explored via the dependence of resistivity on saturation magnetization. This is seen to be approximately exponential (figure 6). This relation, suggested by several authors, works over several decades (nearly six) in resistivity. The data of Garcia Muñoz et al. (1997) can be reexpressed as

$$
\rho(T=0) \simeq \rho_{\text {Mott }} \exp \left\{\lambda\left(\delta M / M_{\mathrm{s}}\right)\right\},
$$

where $\rho_{\text {Mott }} \simeq 2 \mathrm{~m} \Omega \mathrm{cm}, \delta M$ is the deviation of the spontaneous magnetization from the maximum or intrinsic magnetization $M_{\mathrm{s}}$, and $\lambda$ is a large constant, being about 18 for $\mathrm{Sr}$ and about 71 for $\mathrm{Ca}$ (in the family $\left(\mathrm{Re}_{1-y} \mathrm{RE}_{y}^{\prime}\right)_{2 / 3} \mathrm{~A}_{1 / 3} \mathrm{M}_{n} \mathrm{O}_{3}$, where $\mathrm{A}=\mathrm{Sr}$ or $\mathrm{Ca}$ ). The origin of these large factors in the exponent is not clear. The exponential dependence seems to be applicable not only to residual resistivity but also to $\rho(T)$ as a function of thermal equilibrium magnetization $M(T)$ at temperature $T$ (Hundley et al. 1996). In another experiment, Heffner et al. (1996) directly measured the local magnetic field in ferromagnetic $\mathrm{La}_{2 / 3} \mathrm{Ca}_{1 / 3} \mathrm{MnO}_{3}$ by muon spin precession. They again found an exponential relation between $\rho(T)$ and the average muon site internal magnetic field. A Gaussian correlation between $\rho(T)$ and $M(T)$ has been found (O'Donnell et al. 1996) in MBE grown thin films of $\mathrm{La}_{0.7} \mathrm{Ca}_{0.3} \mathrm{MnO}_{3}$. All these latter measurements are, however, over a relatively small, two orders of magnitude range in resistivity.

Colossal magnetoresistance also can be described in terms of the dependence of resistivity on magnetization, as noted by Urushibara et al. (1995). The fact that magnetoresistance is largest near $T_{\mathrm{c}}$ is due to the large magnetic susceptibility there, i.e. the strong dependence of magnetization on magnetic field. At low temperatures and in very clean systems, the magnetization saturates to the maximum possible value, and a magnetic field has only a small effect on it and thus on resistivity. For small values of the magnetization, the resistivity decreases as $M^{2}$.

In addition to atomic level magnetic frustration, multiple grain structure and grain boundary effects could be connected with the high residual resistivity of polycrys-

Phil. Trans. R. Soc. Lond. A (1998) 


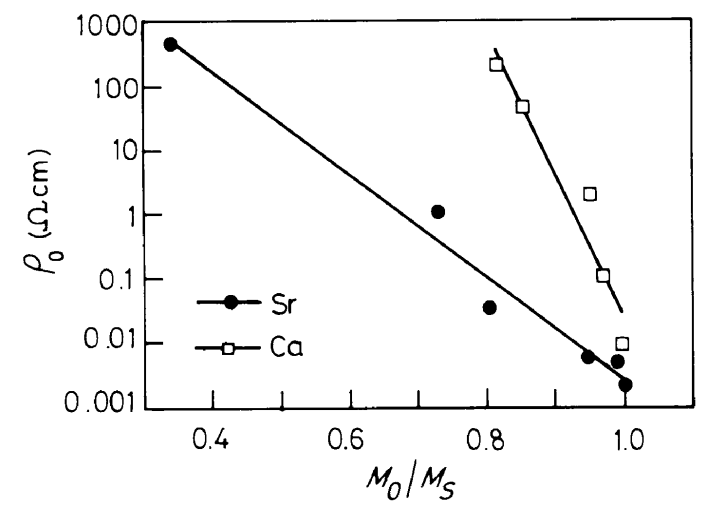

Figure 6 . The residual resistivity $\rho_{0}$ shown in figure 5 , plotted against $T=0$ saturation magnetization $M$ for Sr and Ca ions (from Garcia-Muñoz et al. 1997).

talline ferromagnetic samples (Mahesh et al. 1995a; Gupta et al. 1996). For example, Gupta et al. (1996) found that the residual resistivity of $\mathrm{La}_{2 / 3} \mathrm{Ca}_{1 / 3} \mathrm{MnO}_{3}$ films increases from about $10^{-2}$ to $2 \times 10^{-1} \Omega \mathrm{cm}$ as the grain size decreases from 24 to $3 \mu \mathrm{m}$. Mahesh et al. (1995a) found for a chemically comparable system that, with a further reduction in grain size to $0.02 \mu \mathrm{m}$, the resistivity increases to $320 \Omega \mathrm{cm}$ ! Gupta et al. (1996) analysed their data in terms of a two-medium model with low-resistivity $\left(\rho_{1}\right)$ grains of size $L$ separated by high-resistivity grain boundary layers of thickness $L^{\prime}$ and resistivity $\rho_{2}$. In a simple effective medium theory, the net resistivity is

$$
\rho \cong \rho_{1}+\left(L^{\prime} / L\right) \rho_{2} .
$$

This fits the data reasonably well; the fitting parameter $L^{\prime} \rho_{2}$ is about $6 \times 10^{-5} \Omega \mathrm{cm}^{2}$ (the very small grain data of Mahesh et al. (1995a) suggests a stronger increase in $\rho$ with decreasing grain size). For an interface of thickness $L^{\prime} \simeq 10-12 \AA$, the grain boundary conductance works out to nearly $10^{-5}$ in units of $e^{2} / h$. Such a small conductance for a barrier three atomic layers thick is unusual. Grain boundaries seem to have a very deleterious effect on electron transport. One possiblity could be magnetic frustration induced by grain boundaries on crystallites adjacent to them.

An obvious concern about these results is whether the system is a multiphase mixture, or if it is single phase, what the spatial scale of inhomogeneities is. There is strong X-ray and neutron evidence in many cases that the systems are single phase, even though because of the small ionic radii, there is considerable distortion of the octahedra. Not much is known about the spatial scale of the disorder. Are the systems collections of small grains? What is the nature of the connection between grain size distribution and high residual resistivity? How does the former depend on $\left\langle r_{\mathrm{A}}\right\rangle$ and affect magnetization in the systematic way one sees experimentallly? How can electron transport through a collection of grains and grain boundaries evade the Mott limit at $T=0$ ?

The other possibility is a homogeneous frozen in local disorder due to magnetic frustration, size mismatch, orbital or Jahn-Teller distortion randomness, etc. Again, it is not clear how such disorder can lead to very large residual resistivities.

It does seem from properties of a closely related system with small $\left\langle r_{\mathrm{A}}\right\rangle$, namely single crystal $\mathrm{Pr}_{1-x} \mathrm{Sr}_{x} \mathrm{MnO}_{3}$ with $x=0.3$ and 0.35 (Tomioka et al. 1995; Yoshizawa et al. 1995), that competing interactions that lead to many magnetic phases could

Phil. Trans. R. Soc. Lond. A (1998) 


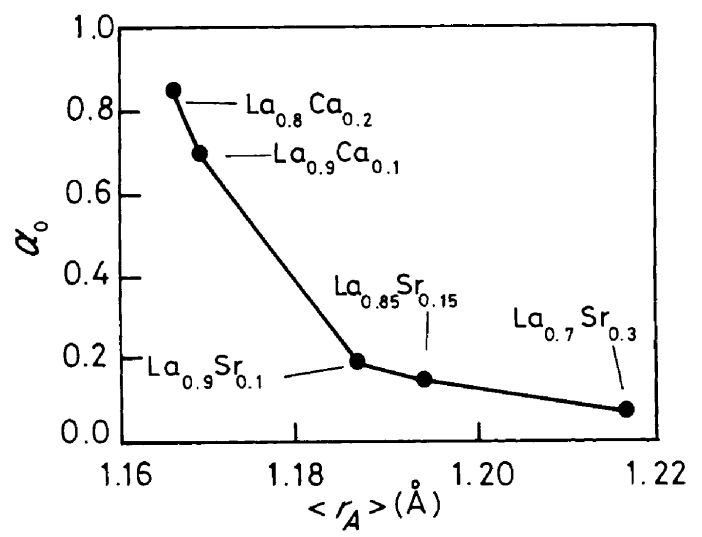

Figure 7. The dimensionless oxygen isotope mass dependence $\alpha_{0}$ of $T_{\mathrm{c}}\left\{\alpha_{0}=\mathrm{d}\left(\ln T_{\mathrm{c}}\right) / \mathrm{d}\left(\ln M_{0}\right)\right\}$ for several manganites, shown as a function of mean A site ion radius $\left\langle r_{\mathrm{A}}\right\rangle$ (from Zhao et al. 1996).

also cause electron localization. A relatively small magnetic field turns this system into a ferromagnetic metal.

\section{Fluctuation effects}

We collect here some observations which suggest clearly that spatial and temporal fluctuations in magnetic moments (direction, size) and lattice ion displacements play an important role in the properties of manganites.

\section{(a) Oxygen isotope effect on $T_{\mathrm{c}}$}

The ferromagnetic transition temperature $T_{\mathrm{c}}$ depends rather strongly on the oxygen isotope mass (Zhao et al. 1996). Figure 7 shows the isotopic exponent $\left(\mathrm{d} T_{\mathrm{c}} / T_{\mathrm{c}}\right) /(\Delta m / m)=\alpha_{0}$ for different systems. For convenience, $\alpha_{0}$ is plotted as a function of the mean radius $\left\langle r_{\mathrm{A}}\right\rangle$ for several $\mathrm{RE}_{1-x} \mathrm{~A}_{x} \mathrm{MnO}_{3}$ manganites. $\alpha_{0}$ decreases from a large value of nearly one for small $\left\langle r_{\mathrm{A}}\right\rangle(c a .1 .16 \AA)$ to about 0.1 for a large $\left\langle r_{\mathrm{A}}\right\rangle(c a .1 .22 \AA)$. Clearly fluctuations in the oxygen ion position strongly affect $T_{\mathrm{c}}$; $T_{\mathrm{c}}$ is larger for the lighter $\mathrm{O}^{16}$ ion because its oscillation amplitude is larger. Such a dependence is absent in a theory where classical or static Jahn-Teller distortion causes the ferromagnetic transition in combination with double exchange.

However, when quantum (zero point) lattice fluctuations are included in a polaronic theory, the effective $e_{g}$ bandwidth depends strongly (exponentially) on the Debye frequency or the ion mass in the site bound polaron limit. Since the ordering temperature is proportional to the bandwidth in the double exchange model, the strong oxygen mass dependence of $T_{\mathrm{c}}$ is argued to point towards the existence of Jahn-Teller polaron (Tomioka et al. 1995; Yoshizawa et al. 1995). At present, there are no estimates of the $\Delta T_{\mathrm{c}} / T_{\mathrm{c}}$ expected, and of its strong dependence on $\left\langle r_{\mathrm{A}}\right\rangle$. (Due to variation in the bond angle? Effective electron phonon coupling?)

\section{(b) $\mu s r$ broadening}

The muon spin precession spectrum shows broadening which is more common in magnetic glasses. For example, Heffner et al. (1996) found that in $\mathrm{La}_{2 / 3} \mathrm{Sr}_{1 / 3} \mathrm{MnO}_{3}$, the muon spin orientation changes with time in a way unlike that of a decaying a particle precessing in a uniform magnetic field. It has, instead of an exponential 
time dependence, a dependence of the form $G_{2} \sim A_{2} \exp \left[-(\Lambda t)^{k}\right]$, where $k$ decreases rapidly from about unity above $T_{\mathrm{c}}(c a .260 \mathrm{~K})$ and close to it, to about 0.5 or so within a range of 40 degrees or so $(250 \mathrm{~K}<T<300 \mathrm{~K})$. Further, the characteristic rate $\Lambda$ shows a rather sharp peak at about $260 \mathrm{~K}$. This implies that within the lifetime of the $\mu$ meson ( $c a \cdot 10^{-6} \mathrm{~s}$ ), the magnetic field at muon site fluctuates. There could also be effects due to variations in the magnitude of the local field. The dynamics of coupled Mn spins is not Debye like; such slow relaxation behaviour is characteristic of glassy systems with localized slowly relaxing configurations and not ferromagnets in the magnetically ordered phase. Another commonly postulated origin of stretched exponential behaviour is a broad distribution of internal fields. Since the magnetic transition is sharp, this does not seem likely. It seems that the coupling between electronic motion and thermally fluctuating spin arrangement gives rise to very slow glassy dynamics.

There is evidence from inelastic neutron scattering that high-frequency spin waves are anomalously broadened at temperatures close to $T_{\mathrm{c}}$.

\section{(c) Optical conductivity}

The optical conductivity of doped manganites reveals an unusual and poorly understood excitation spectrum. Figure 8 shows the real part of the optical conductivity $\sigma(\omega)$ for metallic single crystal $\mathrm{La}_{1-x} \mathrm{Sr}_{x} \mathrm{MnO}_{3}$ with $x=0.3$, as a function of temperature, in an energy range $\hbar \omega \sim 2 \mathrm{eV}$ (Okimoto et al. 1997). At low temperatures, well below $T_{\mathrm{c}}$, there is a roughly Lorentzian structure centred around $\omega=0$, with the conductivity increasing again to a broad peak at round $1 \mathrm{eV}$. Fitting it with a Drude or Lorentz form consistent with the measured DC conductivity leads to a relaxation rate $\hbar / \tau \sim 0.02 \mathrm{eV}$ (rather small) and a Drude number of 0.014 (i.e. $n_{\mathrm{eff}} \sim 0.014 n$ if $m$ is assumed to have the free-electron value and $n$ is the nominal chemical formula carrier density). A more assumption-free estimate of $n_{\mathrm{eff}}$ is obtained from the conductivity sum rule, in which the measured $\sigma(\omega)$ is integrated upto a cut-off $\omega_{c}$. In this case, the cut-off is taken to be the minimum of $\sigma(\omega)$, i.e. one neglects the oscillator strength in the high-frequency peak (this is not very large at low $T$ ). The corresponding carrier density is $c a .0 .03 n$ in the free-electron approximation. Thus there is an enormous depletion (by a factor of 70-30, depending on the rather rough mode of analysis) in the ratio $\left(n / m^{*}\right)$. This is not due to effective mass enhancement, since the specific heat $\gamma$ shows no signs of it, being not far from the value inferred in a free-electron picture. In the ferromagnetic ground state, with resident spins fully aligned, one expects the $e_{g}$ electron to move coherently, more or less like a free particle. This does not seem to happen. Further, the sum rule weight decreases very rapidly with increasing temperature, even in the temperature range where the saturation magnetization does not change much. In a tight binding model, the conductivity sum rule is related to the mean kinetic energy. This is anomalously small.

As temperature increases, there is a dramatic transfer of spectral weight from low frequencies $(c a .0 .5 \mathrm{eV})$ to high frequencies $(0.5 \mathrm{eV}<\hbar \omega<2 \mathrm{eV})$, and above $T_{\mathrm{c}}$ the absorption is peaked at about $0.9 \mathrm{eV}$. This can perhaps be identified with a transition between bands split by the Hund's rule coupling. There is, however, no theoretical understanding of most of the unusual features mentioned above, especially the small low-energy oscillator strength, and the transfer of spectral weight over a large frequency range as a function of temperature. It is possible that the large

Phil. Trans. R. Soc. Lond. A (1998) 


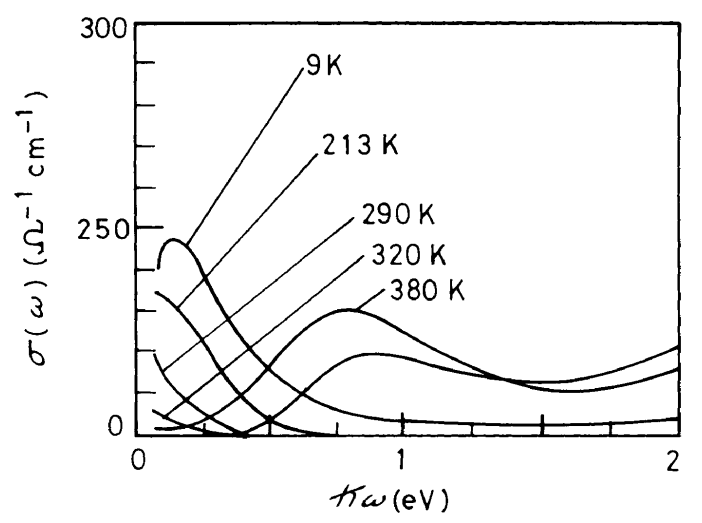

Figure 8. Real part of optical conductivity $\sigma(\omega)$ as a function of frequency $\hbar \omega$, for $\operatorname{La}_{0.7}$ $\mathrm{Sr}_{0.3} \mathrm{MnO}_{3}$ single crystals (from Okimoto et al. 1997). The temperature independent background and phonon contributions have been removed.

temperature-independent incoherent background that grows with $\omega$ is also to be included in the sum rule. The nature of this background is not very clear.

\section{Charge ordering}

It is well known that near the equiatomic composition $\mathrm{RE}_{1 / 2} \mathrm{~A}_{1 / 2} \mathrm{MnO}_{3}$, where $\mathrm{RE}$ is a rare earth ion and $\mathrm{A}$ is a divalent alkaline earth, the $\mathrm{Mn}^{3+}$ and $\mathrm{Mn}^{4+}$ ions, which are nearly equal in concentration, order in space (Tomioka et al. 1995). Depending on the sizes of the $\mathrm{RE}$ and $\mathrm{A}$ ions, a rich variety of phases, e.g. paramagnetic insulator, ferromagnetic metal, charge-ordered paramagnetic insulator, antiferromagnetic insulator, has been observed (Rao et al. 1998; Coey \& Molnar 1998). One of the earliest observations (Tomioka et al. 1995a,b; Yoshizawa 1995) is of the transition from a paramagnet to a ferromagnetic metal to a charge-ordered antiferromagnetic insulator in $\mathrm{Pr}_{1 / 2} \mathrm{Sr}_{1 / 2} \mathrm{MnO}_{3}$ which has a large $\left\langle r_{\mathrm{A}}\right\rangle \simeq 1.24 \AA$ and thus little lattice distortion. The magnetic field temperature phase diagram is shown in figure 9 . A remarkable feature of the transition is the singular effectiveness of the magnetic field in suppressing charge ordering. From the transition temperature $T_{\mathrm{c}} \sim 150 \mathrm{~K}$, one might naievely conclude that the zero temperature energy difference between the two phases (ferromagnetic metal and antiferromagnetic insulator) is about $k_{\mathrm{B}} T_{\mathrm{c}} \sim 150 \mathrm{~K}$, which is the balance between kinetic energy loss due to $e_{g}$ carrier localization in the antiferromagnetic phase, and the coulomb or Madelung energy gain on charge ordering. (The Madelung energy is large, but differences in coulomb energy between a homogeneous metallic mixed valent fluid, namely the ferromagnet, and a valence or charge-ordered solid may not be that large.) However, the magnetic field required to stabilize the ferromagnetic phase at $T=0$ is only $6 T$ or an energy $3.5 \mu_{\mathrm{B}} H \simeq 15 \mathrm{~K}$. This is a factor of ten less than $k_{\mathrm{B}} T_{\mathrm{c}} \simeq 150 \mathrm{~K}$. From single-particle tunnelling, the energy gap in the antiferromagnetic state is about $0.3 \mathrm{eV}$ (Biswas et al. 1997), which is more than an order of magnitude higher than $k_{\mathrm{B}} T_{\mathrm{c}}$ ! Thus, while the qualitative explanations for the possibility of various phases appear reasonable, energy scales which ought to be comparable differ by orders of magnitude.

In general, near equiatomic composition, depending on small changes in the ion size or $\left\langle r_{\mathrm{A}}\right\rangle$, there is a variety of magnetic and charge-ordered phases with dramatically

Phil. Trans. R. Soc. Lond. A (1998) 


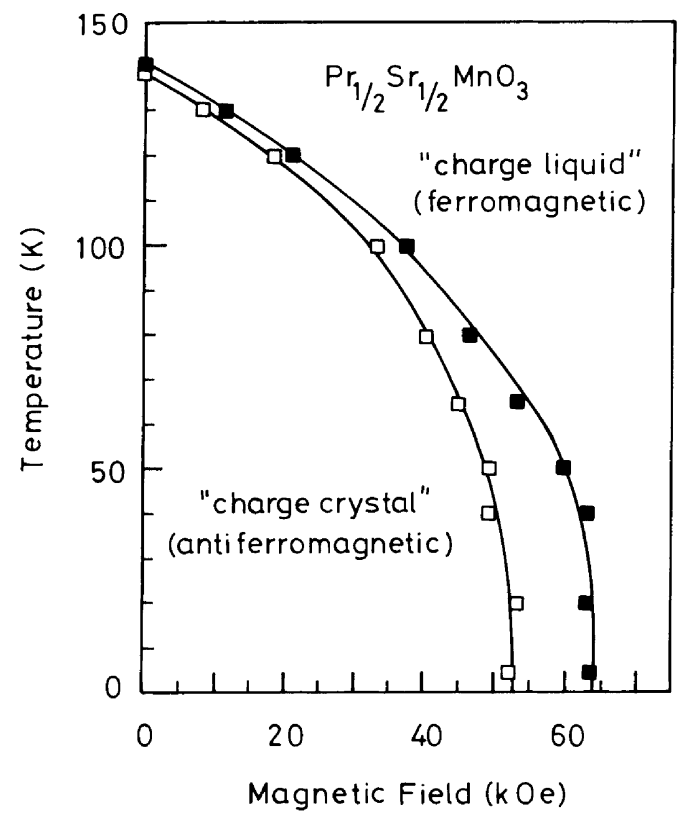

Figure 9. Temperature magnetic field phase diagram of $\operatorname{Pr}_{1 / 2} \mathrm{Sr}_{1 / 2} \mathrm{MnO}_{3}$ (from Tomioka et al. 1995). The full squares represent the transition on heating, and the empty squares the transition on cooling.

different electrical properties (Rao et al. 1998; Coey \& Molnar 1998). This extreme sensitivity of electronic behaviour to small local size change is unique.

\section{Theories}

Against the background of the above phenomena, we outline some of the theories and ideas which have been proposed for this class of systems. Some of the ingredients have been mentioned earlier: strong Hund's rule coupling, large local correlation, Jahn-Teller distortion, dp hybridization, superexchange, various kinds of disorder having to do, for example, with ions of different charges (coulomb disorder) and sizes (local bond distortions). This is a long list, so that several types of theories have been suggested and explored, depending on the factors believed to be crucial.

One extreme approach is to emphasize double exchange and neglect everything else. The resulting frerromagnetic Kondo model (mobile electron spin $\boldsymbol{s}_{i}$ coupled to resident spin $\boldsymbol{S}_{i}$ via a ferromagnetic coupling $\left.-J_{\mathrm{H}} \sum_{i} \boldsymbol{S}_{i} \cdot \boldsymbol{s}_{i}\right)$ has been discussed by Furukawa $(1994,1995 a, b)$ in a single site large $d$, dynamical mean field theory. This model qualitatively reproduces the observed resistivity as a function of magnetization; mainly the initial quadratic part. There is a paramagnet to ferromagnet transition in the model, but both phases are necessarily metallic; the high-temperature phase is a dynamically incoherent electron metal. Some manganites are 'metallic' in the paramagnetic state, though the resistivities are much higher than $\rho_{\text {Mott }}$ (e.g. $\mathrm{La}_{2 / 3} \mathrm{Sr}_{1 / 3} \mathrm{MnO}_{3}$ ), whereas most (e.g. $\mathrm{La}_{2 / 3} \mathrm{Ca}_{1 / 3} \mathrm{MnO}_{3}$ ) are insulators with a rather small activation energy (ca. $0.1 \mathrm{eV}$ ) (Urushibara et al. 1995). The low temperature, as well as residual resistivity behaviour, discussed in $\S 2$ is not understandable within such a model. The fluctuation effects, e.g. isotopic mass dependence of $T_{\mathrm{c}}$, and slow spin dynamics should not exist in this picture. The optical conductivity $\sigma(\omega)$, as

Phil. Trans. R. Soc. Lond. A (1998) 
calculated in simple approximations, is qualitatively similar to that observed at low temperatures. However, the observed anomalously low Drude density is not present in the theory, nor is the 'insulating' spectral density for $T>T_{\mathrm{c}}$. A Drude-like peak is present at all temperatures, as also is a non-zero frequency peak.

In the limit of large Hund's rule coupling $J_{\mathrm{H}}$, an electron can hop from a site $i$ to a site $j$ only if its spin is along the resident classical spin direction so that the bare hopping amplitude $t_{i j}$ is multiplied by projection factors $P_{i} P_{j}$, where $P_{i}=\frac{1}{2}\left(1+\boldsymbol{s}_{i} \cdot \boldsymbol{S}_{i}\right)$. This is the double exchange process (Zener 1951; Anderson \& Hasegawa 1955). We notice here the way in which the electron kinetic energy, its spin direction and the background spins $\left\{\boldsymbol{S}_{i}\right\}$ are dynamically coupled, influencing each other. It has been argued qualitatively (Goodenough 1996) and semi-quantitatively (Varma 1996) that if the background spins are disordered, the electron hopping amplitude can be sufficiently random, so that electronic states near Fermi energy are Anderson localized and the system is an insulator. At lower temperatures, the resident spins are ordered, and electronic states are extended. Thus the paramagnetic to ferromagnetic transition is an Anderson delocalization transition. However, there is as yet no detailed discussion of the quantum many body statistical mechanics of this one parameter model. For example, the resident spin disorder is annealed, i.e. spin configurations change with time, so that the electron hopping randomness is not fixed. Can electronic states still be localized, as they are in liquid semiconductors? How adiabatic is the resident spin dynamics? Can this model, without Jahn-Teller coupling, describe the large changes in electronic spectrum and transport with temperature and electron density.

A complete model for the manganites must include the effect of Jahn-Teller coupling, namely the splitting of the degenerate $e_{g}$ orbital by a local distortion of the oxygen octahedron. The Jahn-Teller splitting is known to be large in the manganites. A model with double exchange $J_{\mathrm{H}} \rightarrow \infty$, and Jahn-Teller coupling between $e_{g}$ orbital and local lattice degrees of freedom has been discussed extensively by Millis, Shraiman and co-workers (Millis et al. 1995, 1996a,b). In this model, the two degenerate orbitals at a site $i$ are coupled by a lattice displacement $\xi$ with strength $\lambda$. Such a displacement costs an elastic energy proportional to $\xi_{i}^{2}$. This full model is difficult to solve. A mean-field theory useful for strong, as well as weak, coupling $\lambda$, but describing only on-site electron dynamics (resulting from the hopping out of and into that site) has been used to analyse the physics.

The qualitative behaviour of the model depends on the effective electron lattice coupling $g=\left(\lambda^{2} / W_{\text {eff }}\right)$, and on the electron filling $n$ (number of electrons per site). Here the effective bandwidth $W_{\text {eff }}$ is large if resident spins are ordered and small if they are not. Since $W_{\text {eff }}$ can be a function of temperature, increasing with decreasing temperature, thermal phase transitions from PM $\rightarrow$ FM, PI $\rightarrow$ FM and PI $\rightarrow$ FI can occur as $\lambda$ increases. (Here $\mathrm{P}$ and $\mathrm{F}$ mean paramagnetic and ferromagnetic, and $\mathrm{M}$ and I mean metallic and insulating.) The qualitative features broadly describe the variety of transitions found in the manganites.

The insulating phase in the approximation used is due to self-consistent small Jahn-Teller polaron formation. The polaronic state drops below the band of extended states, and can form if $g$ is large enough. One major question is whether this is the main mechanism of electron localization, or whether short range magnetic correlations and Anderson localization like effects are also relevant. The large $d$ single site approach used is inadequate for addressing this question, though Millis et al. (1995, $1996 a, b)$ argue that other localizing effects could be small.

Phil. Trans. R. Soc. Lond. A (1998) 
The qualitative correspondance with broad observed features is best for $n=1$ (which in real life is always an insulator). Away from half filling, in the static deformation picture used, one has a certain fraction $(1-n)$ of sites without deformation, and a fraction $n$ which are distorted. This is an instantaneous picture; the real system is homogeneous. The calculated properties describe a metal at all temperatures, or at least a system in which the insulating behaviour is much less evident, because away from half filling, in the approximation used, there is always electronic spectral weight at the Fermi energy.

Many of the phenomena mentioned earlier, e.g. the large residual resistivities, the extreme sensitivity of transport and equilibrium properties to small magnetic fields, the large discrepancy between optical properties and the linear specific heat in terms of a quasi-particle picture, the large isotope effect on $T_{\mathrm{c}}$, are not adequately described in the approximate solutions available of the double exchange model with Jahn-Teller coupling.

It appears, overall, that while we have the ingredients for a reasonably realistic electronic model for the manganites, their consequences are not fully worked out. The available strong coupling but single site dynamical mean field approaches may not be adequate, and may be missing out a new localizing process. Such a process could be responsible for the extreme variation in structures, phases and electronic properties caused by small changes in atomic parameters.

\section{References}

Anderson, P. W. \& Hasegawa, H. 1955 Phys. Rev. 100, 675.

Biswas, A., Raychaudhuri, A. K., Mahendiran, R., Guha, A., Mahesh, R. \& Rao, C. N. R. 1997 J. Phys. Cond. Matt. 9 L355.

Coey, J. M. D. \& von Molnar, S. 1998 Adv. Phys. (In the press.)

Coey, J. M. D., Viret, M., Ranno, L. \& Oundajela, K. 1995 Phys. Rev. Lett. 75, 3910.

Furukawa, N. 1994 J. Phys. Soc. Japan 6, 3214.

Furukawa, N. 1995a J. Phys. Soc. Japan 64, 2734.

Furukawa, N. $1995 b$ J. Phys. Soc. Japan 64, 2754.

Garcia-Munoz, J. L. et al. 1997 Phys. Rev. B 55, R668.

Goodenough, J. B. 1996 MRS Conf. Proc., Boston.

Gupta, A. et al. 1996 Phys, Rev. B 54, R15 629.

Hamilton, J. J., Keatley, E. L., Ju, H. L., Raychaudhuri, A. K., Smolyaninova, V. N. \& Greene, R. L. 1996 Phys. Rev. B 54, 14926.

Heffner, R. H. et al. 1996 Phys. Rev. Lett. 77, 1869.

Hundely, M. F. et al. 1996 J. Appl. Phys. 9, 4535.

Kubo, K. \& Ohata, N. 1972 J. Phys. Soc. Japan 33, 21.

Mahesh, R., Mahendiran, R., Raychaudhuri, A. K. \& Rao, C. N. R. 1995a Appl. Phys. Lett. 68, 2291.

Mahesh, R., Mahendiran, R., Raychaudhuri, A. K. \& Rao, C. N. R. $1995 b$ J. Solid State Chem. 12, 204.

Millis, A. J., Littlewood, P. J. \& Shraiman, B. 1995 Phys. Rev. Lett. 74, 5144.

Millis, A. J., Mueller, R. \& Shraiman, B. $1996 a$ Phys. Rev. B 54, 5389.

Millis, A. J., Mueller, R. \& Shraiman, B. $1996 b$ Phys. Rev. B 54, 5405.

O’Donnell, J., Onellion, M., Rzchowski, M. S., Eckstein, J. N. \& Bozovic, I. 1996 Phys. Rev. B 54, R6841.

Okimoto, Y. et al. 1997 Phys. Rev. B 55, 4206.

Phil. Trans. R. Soc. Lond. A (1998) 
Rao, C. N. R., Mahesh, R., Raychaudhuri, A. K. \& Mahendiran, R. 1998 J. Phys. Chem. Solids. Snyder, G. J., Hiskes, R., DiCarolis, S., Beasley, M. R. \& Geballe, T. H. 1996 Phys. Rev. B 53, 1443.

Tomioka, Y. et al. 1995 J. Phys. Soc. Japan 64, 3626.

Tomioka, Y., Asamitsu, A., Moritomo, Y., Kuwahara, H. \& Tokura, Y. 1996 Phys. Rev. Lett. $\mathbf{7 4}, 5108$.

Urushibara, A. et al. 1995 Phys. Rev. B 51, 14103.

Varma, C. M. 1996 Phys. Rev. B 54, 7328.

Viret, M., Ranno, L. \& Coey, J. M. D. 1997 Phys. Rev. B 55, 8067.

Yoshizawa, H. et al. 1995 Phys. Rev. B 52, R13 145.

Zener, C. 1951 Phys. Rev. 82, 403.

Zhao, G. M., Conder, K., Keller, H. \& Muller, K. A. 1996 Nature 381, 676.

\section{Discussion}

J. E. Enderby (Department of Physics, University of Bristol, UK). The grain boundary resistance seems to be a consequence of quantum confinement, which must occur at length scales of $c a$. $10 \AA$. It would seem that the level spectra were such as to make the transmission coefficient very small.

T. V. Ramakrishnan. The electrons are in the grains which are large, about a micron or so in linear dimension. In the absence of degeneracies, the level spacing would be very small, ca. $10^{-10} \mathrm{eV}$ or so. These grains are weakly coupled through the (ca. $10 \AA$ thick) insulating barriers. Quantum confinement effects are not likely under these conditions.

R. L. Johnston (School of Chemistry, University of Birmingham, UK). I would like to follow up the point made by Professor Enderby. Extrapolation from the bulk to finite grain size is analogous to the application of the 'liquid drop model' to clusters. It is known that this approximation breaks down for nm-sized clusters due to quantum size effects (non-smooth variation of properties with size).

T. V. RAMAKRishnan. The grains in these systems are micron sized, so quantum size effects should not be serious at accessible temperatures. (Energy level specing is approximately $10^{-10} \mathrm{eV}$ in the absence of degeneracies.)

A. S. Alexandrov (Department of Physics, Loughborough University of Technology, $U K)$. I am pleased to hear that small polarons are relevant for GMR. However, there is a problem with the terminology 'Jahn-Teller polaron'. The activation energy seen in the resistivity seems to be one order of magnitude smaller than that expected from a Jahn-Teller distortion.

T. V. RAMAKRISHNan. It is true that the activation energy for resistivity is about $0.1 \mathrm{eV}$ whereas in the site localized limit, the Jahn-Teller binding energy can be about $1 \mathrm{eV}$ or more. This probably means that within the $\mathrm{J}-\mathrm{T}$ picture, one is not in the site localized limit and that quantum fluctuations are important. These have been considered partly be several authors, who find a considerable reduction in the activation energy with respect to the site localized limit.

H. Von LöHneysen (Physikalisches Institut, Universitat Karlsruhe, Germany). Is there any relation between the prefactor of the low-temperature $T^{2}$ resistivity and the linear specific-heat coefficient $\gamma$, as expected in a Fermi-liquid approach where the $T^{2}$ resistivity arises from electron-electron interactions?

Phil. Trans. R. Soc. Lond. A (1998) 
T. V. RAMAKRishnan. One of the strange things about the $T^{2}$ term is that there is no such relation. The $T^{2}$ coefficient is highly enhanced, but the specific-heat coefficient is appropriate to a d band metal without enhancement.

P. Coleman (Department of Physics and Astronomy, Rutgers University, USA). In the discussion of grain-boundary resistance was the fitting parameter, $L, 10^{-2} \AA$, or was it $10^{2} \AA$ ?

T. V. RAMAKRishnan. It was $10^{-2} \AA$. I was trying to make the point that if one naively assumes the validity of the two medium formula (equation (3.1)) and attempts to fit the observed large residual resistivities with it, one reaches the absurd conclusion that both metallic grains ought to be $10^{-2} \AA$ or so in size. The effective medium theory may be inapplicable, or the physical picture of small grains and grain boundaries as the cause of high residual resistivity may be appropriate.

R. L. McGreevy (Studsvik Neutron Research Laboratory, Sweden). Dr Ramakrishnan mentioned the possible importance of correlations between individual atomic positions and spins. The importance of disorder in perovskites has also been stressed. I would like to point out that our typical view of disorder is an 'average' view; locally, there may be large fluctuations in correlations between individual atoms. This can lead to a dramatic change in macroscopic properties, e.g. conductivity, being caused by small and continuously varying correlations (e.g. as a function of composition). A good analogue is with the melting transition where there is first-order transition in terms of long-range order, and a dramatic change in, for example, viscosity, but the short-range order changes very little.

T. V. RAmakrishnan. It is quite possible that statistical fluctuations in the local spin arrangement or $e_{g}$ orbital admixture can cause major changes in DC transport behaviour.

Y. IYE (ISSP, University of Tokyo, Japan). Dr Ramakrishnan showed that there is a huge change in the residual resistivity by changing the grain size. Am I right in understanding that it does not affect the $T^{2}$ term?

T. V. RAMAKRishnan. I do not know.

C. N. R. RaO (Indian Institute of Science, Bangalore, India). With reference to Coey's data that Dr Ramakrishnan showed, I would like to point out that large residual resisitivities are generally found in manganates with small $\left\langle r_{\mathrm{A}}\right\rangle$ or bad A ion mismatch. Furthermore, it is only at very small $\left\langle r_{\mathrm{A}}\right\rangle$ that we find really chargeordered systems driven by cooperative Jahn-Teller effect (long-range elastic strain).

T. V. Ramakrishnan. Yes. Actually, these two observations may be connected, namely large residual resistivities may be due to large $e_{g}$ orbital order-disorder effects. 
